\section{BMJ Open Respiratory Research}

\title{
Predictive value of prebronchodilator and postbronchodilator spirometry for COPD features and outcomes
}

\author{
Spyridon Fortis, ${ }^{1,2}$ Michael Eberlein, ${ }^{1}$ Dimitris Georgopoulos, ${ }^{2,3}$ \\ Alejandro P Comellas ${ }^{1}$
}

To cite: Fortis S, Eberlein M, Georgopoulos D, et al. Predictive value of prebronchodilator and postbronchodilator spirometry for COPD features and outcomes. BMJ Open Resp Res 2017;4:e000213. doi:10.1136/ bmjresp-2017-000213

Received 11 May 2017 Revised 17 October 2017 Accepted 7 November 2017
CrossMark

${ }^{1}$ Division of Pulmonary, Critical Care and Occupational Medicine, University of lowa Hospitals and Clinics, lowa City, Iowa, USA

${ }^{2}$ Medical School, University of Crete, Heraklion, Greece ${ }^{3}$ Departments of Pulmonary Medicine and Intensive Care Medicine, University Hospital of Heraklion, Heraklion, Greece

Correspondence to Dr Spyridon Fortis; spyridon-fortis@uiowa.edu

\section{ABSTRACT}

Introduction We compared the predictive value of prebronchodilator and postbronchodilator spirometry for chronic obstructive pulmonary disease (COPD) features and outcomes.

Methods We analysed COPDGene data of 10192 subjects with smoking history. We created regressions models with the following dependent variables: clinical, functional and radiographic features, and the following independent variables: prebronchodilator airflow obstruction (PREO) and postbronchodilator airflow obstruction (POSTO), prebronchodilator and postbronchodilator $\mathrm{FEV}_{1} \%$ predicted. We compared the model performance using the Akaike information criterion (AIC).

Results The COPD prevalence was higher using PRE0. About $8.5 \%$ had PRE0 but no airflow obstruction in postbronchodilator spirometry (POSTN) (PREO-POSTN) and $3 \%$ of all subjects had no aiflow obstruction in prebronchodilator spirometry (PREN) but POSTO (PRENPOSTO). We found no difference in COPD features and outcomes between PREO-POSTN and PRENPOSTO subjects. Although, both prebronchodilator and postbronchodilator spirometries are both associated with chronic bronchitis, dyspnoea, exercise capacity and COPD radiographic findings, models that included postbronchodilator spirometric measures performed better than models with prebronchodilator measures to predict these COPD features. The predictive value of prebronchodilator and postbronchodilator spirometries for respiratory exacerbations, change in forced expiratory volume in $1 \mathrm{~s}$, dyspnoea and exercise capacity during a 5 -year period is relatively similar, but postbronchodilator spirometric measures are better predictors of mortality based on AIC.

Conclusions Postbronchodilator spirometry may be a more accurate predictor of COPD features and outcomes.

\section{INTRODUCTION}

Chronic obstructive pulmonary disease (COPD) diagnosis is based on a spirometric definition according to Global Initiative for Chronic Obstructive Lung Diseases guidelines. ${ }^{1}$ This diagnosis requires the presence of airflow obstruction (AFO) defined as forced expiratory volume in $1 \mathrm{~s}\left(\mathrm{FEV}_{1}\right) /$ forced vital capacity (FVC) below the lower limit of normal (LLN) or $0.7 .{ }^{1}$ Several studies

\section{Key messages}

The chronic obstructive pulmonary disease (COPD) prevalence was higher using prebronchodilator spirometry.

- We found no difference in COPD features and outcomes between subjects with discordance in prebronchodilator and postbronchodilator spirometry.

- Although both prebronchodilator and postbronchodilator spirometries are associated with COPD features and outcomes, postbronchodilator spirometry may be a more accurate predictor.

have compared $\mathrm{FEV}_{1} / \mathrm{FVC}<\mathrm{LLN}$ with $\mathrm{FEV}_{1} /$ $\mathrm{FVC}<0.7$ as a diagnostic criterion for $\mathrm{AFO} .^{2-4}$ GOLD recommends a postbronchodilator $\mathrm{FEV}_{1} / \mathrm{FVC}<0.7$. The rationale is based on its simplicity, independence of reference values and the fact that it is used in numerous clinical trials. ${ }^{1}$ However, to our knowledge, there is little evidence to support the use of postbronchodilator spirometry for COPD diagnosis over prebronchodilator spirometry. A single study with 300 subjects showed that postbronchodilator spirometry is a better predictor of mortality than prebronchodilator spirometry in COPD. ${ }^{5}$ Mannino et at showed that both prebronchodilator and postbronchodilator lung function predict mortality with a similar accuracy.

COPD prevalence is lower when postbronchodilator spirometry is used compared with when prebronchodilator spirometry is used. ${ }^{7}$ In the absence of a true 'gold standard' for COPD diagnosis, the utility of a diagnostic test depends on whether it can predict outcomes or change disease management. Previous studies showed conflicting results regarding the ability of prebronchodilator and postbronchodilator spirometry in predicting mortality. ${ }^{5}{ }^{6}$ Whether postbronchodilator spirometry is superior to prebronchodilator spirometry to predict outcomes other than 
mortality has not been studied. We hypothesised that prebronchodilator and postbronchodilator spirometries are associated with COPD features and predict outcomes with the same accuracy.

Subjects with significant hyperinflation, and therefore with more dyspnoea, ${ }^{8}$ may have a reduced prebronchodilator $\mathrm{FVC}$ and a normal $\mathrm{FEV}_{1} / \mathrm{FVC}$ ratio. ${ }^{9}$ Because FVC increase is more common than $\mathrm{FEV}_{1}$ increase after bronchodilator, ${ }^{10}$ postbronchodilator $\mathrm{FEV}_{1} / \mathrm{FVC}<0.7$ (POSTO) may be more sensitive to diagnose symptomatic patients with clinically significant hyperinflation than prebronchodilator $\mathrm{FEV}_{1} / \mathrm{FVC}<0.7$ (PREO) ${ }^{11}$ Therefore, it is critical to examine whether subjects with PREO and no $\mathrm{AFO}$ in postbronchodilator spirometry (POSTN) would have more clinical, functional and radiographic COPD features than subjects with POSTO and no AFO in prebronchodilator spirometry (PREN). Do clinical, functional and radiographic features differ between subjects with PREO and POSTN and subjects with PREN and POSTO?

We compared the predictive value of prebronchodilator and postbronchodilator $\mathrm{FEV}_{1} / \mathrm{FVC}<0.7$ and $\mathrm{FEV}_{1} \%$ predicted for chronic bronchitis, dyspnoea, exercise capacity and COPD radiographic findings at phase 1 (baseline); respiratory exacerbations, change in dyspnoea, $\mathrm{FEV}_{1}$ and exercise capacity from phase 1 to phase 2 visit (about 5 years apart); and mortality. We also examined whether subjects with discordance in prebronchodilator and postbronchodilator spirometry have different clinical, functional and radiographic features.

\section{METHODS}

\section{Data collection}

We conducted the study using data from the COPDGene database. GOPDGene is an ongoing study that enrolled subjects in several clinical centres through the USA (http://www.copdgene.org/). The institutional review boards at each participating centre approved the study protocol. Details of the study protocol have been published previously. ${ }^{12}$ Briefly, all subjects provided informed consent before participation in the study. Subjects are self-identified non-Hispanic whites or African-Americans between the ages of 45 and 80 years. They completed a modified American Thoracic Society Respiratory Epidemiology Questionnaire, St. George's Respiratory Questionnaire (SGRQ) and 6 min walk test at phase 1 visit (baseline). Subjects performed prebronchodilator and postbronchodilator spirometry according to American Thoracic Society-European Respiratory Society (ATS-ERS) guidelines. ${ }^{13}$ After prebronchodilator spirometric manoeuvres, two puffs of albuterol metereddose inhaler were administered using a spacer. Postbronchodilator manoeuvres were performed between 15 and $40 \mathrm{~min}$ after albuterol administration and preferably between 15 and $20 \mathrm{~min}$. Subjects performed inspiratory and expiratory chest CT scans using multidetector CT scanners as per protocol. ${ }^{12}$ Volumetric CT scans were obtained at maximal inspiration (total lung capacity (TLC)) and end-tidal expiration (functional residual capacity (FRC)). Emphysema and gas trapping were quantitated using 3D Slicer software (www.airwayinspector.org), and airway dimensions were measured using Pulmonary Workstation 2 (VIDA Diagnostics, Coralville, Iowa, USA). ${ }^{12}$

We included all subjects who participated in COPDGene study with at least 10 or more pack-years of smoking and who completed a phase 1 visit $(n=10192)$. Subjects were contacted every 6 months and completed a validated questionnaire regarding respiratory exacerbations. About 5 years after the phase 1 visit, a portion of subjects had a phase 2 visit that included questionnaire as in phase 1, a prebronchodilator and postbronchodilator spirometry and $6 \mathrm{~min}$ walk test. We also collected all-cause mortality data. We excluded those with incomplete prebronchodilator and postbronchodilator spirometric data.

\section{Definitions and outcomes}

Prebronchodilator AFO (PREO) was defined as prebronchodilator $\mathrm{FEV}_{1} / \mathrm{FVC}<0.7$. Postbronchodilator $\mathrm{AFO}$ (POSTO) was defined as postbronchodilator $\mathrm{FEV}_{1} / \mathrm{FVC}$ $<0.7$. Prebronhodilator FEV1\% predicted $($ Pre-FEV $\%$ ) and postbronchodilator FEV1\% predicted (post-FEV $\%$ ) were calculated using predicted equations by Hankinson et al. ${ }^{14}$ Bronchodilator response was defined as an increase in $\mathrm{FEV}_{1}$ or $\mathrm{FVC}$ equal to or greater than $0.2 \mathrm{~L}$ and $12 \%$ according to ATS-ERS guidelines. ${ }^{9}$

Emphysema was defined by using the percentage of lung volume at TLC with attenuation less than -950 Hounsfield units (HU). ${ }^{12} 15$ Expiratory CT scans were performed at FRC. Gas trapping was quantified as the percentage of lung volume at FRC with attenuation less than -856 HU. ${ }^{12} 15$

Exacerbations were defined as episodes of worsening respiratory symptoms requiring use of antibiotics and systemic steroids since the phase 1 visit. Severe exacerbations were defined as those requiring hospitalisations. Other variables definitions have been previously described. ${ }^{1215}$

\section{Statistical analysis}

We performed a McNemar test for paired binary data to compare the prevalence rate of AFO using PREO and POSTO as diagnostic criteria.

We stratified the subjects by prebronchodilator and postbronchodilator $\mathrm{FEV}_{1} / \mathrm{FVC}$ to:

- Prebronchodilator $\mathrm{FEV}_{1} / \mathrm{FVC} \geq 0.7$ (PREN) and postbronchodilator $\mathrm{FEV}_{1} / \mathrm{FVC} \geq 0.7(\mathrm{POSTN})=(\mathrm{PREN}-$ POSTN).

- Prebronchodilator $\quad \mathrm{FEV}_{1} / \mathrm{FVC}<0.7 \quad$ (PREO) and postbronchodilator $\quad \mathrm{FEV}_{1} / \mathrm{FVC} \quad<0.7 \quad$ (POSTO) $=($ PREO-POSTO $)$. 
- Prebronchodilator $\mathrm{FEV}_{1} / \mathrm{FVC}<0.7$ (PREO) and postbronchodilator $\mathrm{FEV}_{1} / \mathrm{FVC} \geq 0.7(\mathrm{POSTN})=(\mathrm{PREO}-$ POSTN).

- Prebronchodilator $\mathrm{FEV}_{1} / \mathrm{FVC} \geq 0.7$ (PREN) and postbronchodilator $\mathrm{FEV}_{1} / \mathrm{FVC}<0.7 \quad(\mathrm{POSTO})=$ (PREN-POSTO).

We compared characteristics at the phase 1 visit, number of exacerbations per year, changes in $\mathrm{FEV}_{1}$, dyspnoea score and distance covered in 6 min walk from the phase 1 to the phase 2 visit between PREO-POSTN and PREN-POSTO using Fischer's exact or $\chi^{2}$ test for categorical variables and Student's t-test or Wilcoxon rank sum test for normal and non-normal continuous variables, respectively. When we found a significant difference in the above measures between PREO-POSTN and PRENPOSTO in unadjusted analysis, we created multivariable regression models with PREO-POSTN versus PRENPOSTO as an independent variable.

We performed multivariable logistic regression models with chronic bronchitis at baseline as the dependent variable (outcome) and PREO, POSTO, pre-FEV $\%$ and post-FEV ${ }_{1} \%$ as the independent variables. Similarly, we performed multivariable linear regression models with the following dependent variables: (1) dyspnoea scores, emphysema\%, gas trapping\% and distance covered in 6 min walk at phase $1 ;(2)$ number of respiratory exacerbations and severe exacerbations per year; and (3) changes in $\mathrm{FEV}_{1}$, dyspnoea and distance covered in $6 \mathrm{~min}$ walk between phase 1 and phase 2 visit, and prebronchodilator and postbronchodilator measures as the independent variables.

We used a Cox proportional hazard regression analysis to examine the association of spirometric measures and patterns with mortality.

All regression models included the following covariates: age, sex, race, smoking status, pack-years, history of asthma (per questionnaire), diabetes, high blood pressure, stroke and sleep apnoea. We used the Akaike information criterion (AIC) to compare the performance of the various models. ${ }^{16}{ }^{17}$ Lower AIC by 7 indicates better model performance. ${ }^{16}$ We used $\mathrm{R}$ software package (http://www.r-project.org/) for all statistical analysis.

\section{RESULTS}

Of 10192 subjects with at least 10 or more pack-years of smoking, we excluded 192 with incomplete prebronchodilator and postbronchodilator spirometric data. Ten thousand subjects were included in in the analysis. We had available data regarding respiratory exacerbations for 8479 subjects. Of the 10000 subjects, 4857 completed a phase 2 visit that included respiratory questionnaires, spirometry and $6 \mathrm{~min}$ walk test. We also had available mortality data for 8221 subjects.

Using PREO, the AFO prevalence was 50.2\% (5016 of $10000)$, whereas using POSTO, the prevalence was $44.5 \%$ (4451 of $10000 ; \mathrm{P}<0.001$ ). There were 1167 subjects with discordant spirometry: 866 (8.7\%) with PREO-POSTN and $301(3 \%)$ with PREN-POSTO (supplementary table $1)$.

\section{Baseline characteristics at phase $1(n=10000)$}

Table 1 shows the characteristics of subjects with PREOPOSTO, PREN-POSTN, PREO-POSTN and PRENPOSTO. Compared with PREO-POSTN subjects, PRENPOSTO subjects had higher body mass index, higher prevalence of hypertension, higher dyspnoea scores, lower post-FEV $\%$ and shorter distance covered in $6 \mathrm{~min}$ walk. Bronchodilator response was more common in PREN-POSTO subjects than in PREN-POSTN subjects. Change in $\mathrm{FEV}_{1} \%$ predicted after bronchodilator was smaller, but change FVC\% predicted after bronchodilator was larger in PREN-POSTO subjects compared with subjects with PREO-POSTN.

To investigate further the higher dyspnoea scores and shorter distance covered in $6 \mathrm{~min}$ walk in PREOPOSTN subjects compared with PREN-POSTO subjects, we performed multilinear regression analysis and found that PREN-POSTO is associated with increased dyspnoea and reduced exercise capacity. (supplementary table 2). However, when we added post-FEV $\%$ in the models, this association disappeared.

In the adjusted analysis, both PREO and POSTO were associated with chronic bronchitis, dyspnoea scores, radiographic percent emphysema and gas trapping and distanced covered in $6 \mathrm{~min}$ walk at the phase 1 visit, but based on the AIC, models that included POSTO performed better than models that included PREO to predict those outcomes (table 2). We found similar results for pre- $\mathrm{FEV}_{1} \%$ and post-FEV $\%$.

Respiratory exacerbations ( $n=8479)$ and changes in clinical and functional features at phase $2(n=4857)$

We found no difference in the number of exacerbations and severe exacerbations per year between PREOPOSTO and PREN-POSTO subjects followed in average for $4.5 \pm 1.8$ years (table 3 ). The drop in $\mathrm{FEV}_{1}$ between the phase 1 and 2 visits was greater in PREO-POSTN compared with the change in PREN-POSTO subjects, whereas the change in distance covered in $6 \mathrm{~min}$ walk was smaller in the PREO-POSTN compared with PREN-POSTO group. In adjusted analysis, PREN-POSTO subjects had a smaller reduction in $\mathrm{FEV}_{1}$ compared with PREO-POSTN subjects, but when we adjusted for postbronchodilator $\mathrm{FEV}_{1} \%$ that association disappeared (supplementary table 3).

In multilinear regression analysis, both PREO and POSTO correlated significantly with number of exacerbations and severe exacerbations per year, dyspnoea scores and distance covered in 6 min walk between phase 1 and 2 visits (table 4). Based on AIC, models showed similar performance to predict those outcomes regardless whether PREO or POSTO was included, except models for number of exacerbations and change in SGRQ score, where those models that included POSTO performed better compared with those that included PREO. 
Table 1 Baseline characteristics

\begin{tabular}{|c|c|c|c|c|c|c|}
\hline & $\begin{array}{l}\text { All } \\
(n=10000)\end{array}$ & $\begin{array}{l}\text { PREO-POSTO } \\
(n=4150)\end{array}$ & $\begin{array}{l}\text { PREN-POSTN } \\
(n=4683)\end{array}$ & $\begin{array}{l}\text { PREO-POSTN } \\
(\mathrm{n}=866)\end{array}$ & $\begin{array}{l}\text { PREN-POSTO } \\
(\mathrm{n}=301)\end{array}$ & P value* \\
\hline Age, year $\pm S D$ & $59.6 \pm 9$ & $63.4 \pm 8.5$ & $56.4 \pm 8.2$ & $59.3 \pm 8.9$ & $59.1 \pm 8.9$ & 0.72 \\
\hline Female, \% (n) & $46.7(4668)$ & $43.9(1823)$ & $49.4(2315)$ & $44.6(386)$ & $47.8(144)$ & 0.36 \\
\hline Non-white race, \% (n) & $32.9(3287)$ & 21.6 (895) & $42.9(2010)$ & $31.4(272)$ & 36.5 (110) & 0.12 \\
\hline $\mathrm{BMI}, \mathrm{kg} / \mathrm{m}^{2}{ }^{2} \mathrm{SD}$ & $28.8 \pm 6.3$ & $27.8 \pm 6.1$ & $29.7 \pm 6.3$ & $28.6 \pm 6.3$ & $29.5 \pm 6.4$ & 0.023 \\
\hline$P P Y \pm S D$ & $44.3 \pm 24.9$ & $52.2 \pm 27.2$ & $37.8 \pm 20.8$ & $41.9 \pm 22.8$ & $43.2 \pm 24.3$ & 0.57 \\
\hline Active smoking, \% (n) & $52.8(5279)$ & $42.1(1746)$ & $60.9(2853)$ & $57.4(497)$ & $60.8(183)$ & 0.33 \\
\hline Chronic bronchitis, \% (n) & $19.2(1922)$ & $26.5(1101)$ & $13.3(621)$ & $16.6(144)$ & $18.6(56)$ & 0.48 \\
\hline Asthma, \% (n) & 19.4 (1943) & 25.3 (1049) & $14.4(674)$ & 18.5 (160) & $19.9(60)$ & 0.59 \\
\hline CAD, \% (n) & $6.5(648)$ & $9(375)$ & $4.4(207)$ & $5.4(47)$ & $6.3(19)$ & 0.67 \\
\hline $\mathrm{CHF}, \%(\mathrm{n})$ & 3.2 (320) & $4.6(192)$ & 2 (96) & $2.3(20)$ & $4(12)$ & 0.18 \\
\hline DM, \% (n) & $13(1301)$ & $11.9(494)$ & $13.6(638)$ & $14.2(123)$ & $15.3(46)$ & 0.64 \\
\hline HTN, \% (n) & $43.2(4322)$ & $48.4(2008)$ & $39(1830)$ & 39.7 (344) & $46.5(140)$ & 0.046 \\
\hline OSA, \% (n) & $14.6(1459)$ & $16(665)$ & $13.3(624)$ & $14(121)$ & $16.3(49)$ & 0.39 \\
\hline Stroke, \% (n) & $2.6(258)$ & 3.4 (139) & $1.9(90)$ & $2.1(18)$ & $3.7(11)$ & 0.19 \\
\hline $\mathrm{SaO}_{2}, \% \pm \mathrm{SD}$ & $96.1 \pm 2.9$ & $94.9 \pm 3.5$ & $97 \pm 2.1$ & $96.8 \pm 2.4$ & $96.5 \pm 2.5$ & 0.15 \\
\hline $\mathrm{MMRC}_{ \pm} \mathrm{SD}$ & $1.4 \pm 1.4$ & $1.9 \pm 1.5$ & $0.9 \pm 1.3$ & $1 \pm 1.3$ & $1.3 \pm 1.5$ & 0.0011 \\
\hline$S G R Q \pm S D$ & $27.4 \pm 22.9$ & $37.6 \pm 22.7$ & $19.5 \pm 19.8$ & $21.3 \pm 20.8$ & $27.2 \pm 23.1$ & $<0.001$ \\
\hline Post-FEV $1, \% \pm S D$ & $76.3 \pm 25.6$ & $55.9 \pm 22.4$ & $92.6 \pm 15.4$ & $84.7 \pm 15.9$ & $77.3 \pm 17.4$ & $<0.001$ \\
\hline Post-FVC, $\% \pm S D$ & $86.9 \pm 18.3$ & $81.2 \pm 20.3$ & $91.4 \pm 15.1$ & $89 \pm 16.7$ & $91.6 \pm 18.8$ & 0.087 \\
\hline BDR, \% (n) & $21.5(2146)$ & $33.9(1408)$ & $9.2(432)$ & $19.5(169)$ & 45.5 (137) & $<0.001$ \\
\hline Delta FEV ${ }_{1}, \% \pm S D$ & $5.8 \pm 10.3$ & $8.8 \pm 12.1$ & $3 \pm 6.6$ & $7.7 \pm 10.8$ & $1.5 \pm 15.5$ & $<0.001$ \\
\hline Delta FVC, \% ${ }^{2} \mathrm{SD}$ & $3.9 \pm 12.6$ & $7.5 \pm 13.2$ & $0.9 \pm 7.5$ & $-2.1 \pm 9.3$ & $17.7 \pm 34.4$ & $<0.001$ \\
\hline Emphysema, \% $\pm \mathrm{SD} † \ddagger$ & $7.6 \pm 10.6$ & $14 \pm 12.9$ & $2.2 \pm 2.8$ & $2.9 \pm 3.1$ & $3.4 \pm 5$ & 0.45 \\
\hline Gas trapping, $\% \pm S D \dagger \ddagger$ & $24.2 \pm 20.8$ & $39.3 \pm 20.5$ & $10.6 \pm 9$ & $15 \pm 10$ & $17.1 \pm 12.6$ & 0.19 \\
\hline 6-MWD, feet $\pm S D$ & $1351 \pm 399$ & $1224 \pm 408.2$ & $1443 \pm 365.3$ & $1447 \pm 370.1$ & $1352 \pm 382.3$ & $<0.001$ \\
\hline
\end{tabular}

${ }^{*}$ Comparison between PREO-POSTN and PREN-POSTO using $\chi^{2}$ and Student's t-test or Wilcoxon rank sum test.

†For emphysema and gas trapping analysis, data were available for 5553 and 4945 subjects, respectively.

$\ddagger$ Gas trapping was measured at functional residual capacity.

6-MWD, 6 min walk distance; BDR, bronchodilator response; BMI, body mass index; CAD, coronary artery disease; CHF, congestive heart failure; Delta FEV $\%$, \% change in FEV after bronnchodilator; Delta FVC\%, \% change in FVC after bronchodilator; DM, diabetes mellitus; $\mathrm{FEV}_{1}$, forced expiratory volume in $1 \mathrm{~s}$; FVC, forced vital capacity; HTN, hypertension; MMRC, modified Medical Research Council dyspnoea score; OSA, obstructive sleep apnoea; pre- $\mathrm{FEV}_{1} \%$, prebronchodilator $\mathrm{FEV}_{1} \%$ predicted; post-FEV $\%$, postbronchodilator $\mathrm{FEV}_{1} \%$ predicted; PPY, pack per year; $\mathrm{SaO}_{2}$, arterial oxygen saturation; SGRQ, St. George's Respiratory Questionnaire score;6-MWD = 6-min walk distance. PREN-POSTN: prebronchodilator $\mathrm{FEV}_{1} / \mathrm{FVC}>0.7$ and postbronchodilator $\mathrm{FEV}_{1} / \mathrm{FVC}>0.7$. PREN-POSTO: prebronchodilator $\mathrm{FEV}_{1} / \mathrm{FVC}>0.7$ and postbronchodilator $\mathrm{FEV}_{1} / \mathrm{FVC}<0.7$. PREO-POSTNO: prebronchodilator $\mathrm{FEV}_{1} / \mathrm{FVC}<0.7$ and postbronchodilator $\mathrm{FEV}_{1} / \mathrm{FVC}<0.7$. PREO-POSTN: prebronchodilator $\mathrm{FEV}_{1} / \mathrm{FVC}<0.7$ and postbronchodilator $\mathrm{FEV}_{1} / \mathrm{FVC}>0.7$.

Similarly, both prebronchodilator and postbronchodilator $\mathrm{FEV}_{1} \%$ correlated significantly with the number of exacerbations and severe exacerbations per year, change in $\mathrm{FEV}_{1}$, dyspnoea scores and distance covered in $6 \mathrm{~min}$ walk between phase 1 and 2 visits (table 4). Models showed similar performance except models for number of exacerbations (pre- $\mathrm{FEV}_{1} \%$ models performed better) and for change in $\mathrm{FEV}_{1}$ (pre-FEV $\%$ models performed better).

\section{Spirometric pattern at phase 2}

Of 377 subjects with PREO-POSTN at phase 1 who had both prebronchodilator and postbronchodilator spirometry at the phase 2 visit, the phase 2 spirometry showed
PREO-POSTO in 167 (44.3\%), PREN-POSTN in 120 (31.8\%), PREO-POSTN in $72(19.1 \%)$ and PREN-POSTO in $18(4.8 \%)$ (supplementary table 4$)$. Of those subjects who progressed to PREO-POSTO, 52.1\% were active smokers; only $39.2 \%$ of those who progressed to PRENPOSTN were active smokers at the phase 2 visit $(\mathrm{P}=0.03)$.

Of 166 PREN-POSTO at phase 1 who had both prebronchodilator and postbronchodilator spirometry at the phase 2 visit, the phase 2 spirometry showed PREOPOSTO in $82(49.4 \%)$, PREN-POSTN in $51(30.7 \%)$, PREO-POSTN in $24(14.5 \%)$ and PREN-POSTO in 9 $(5.4 \%)$ (supplementary table 4 ). Of those subjects who progressed to PREO-POSTO, $50 \%$ were active smokers, 
Table 2 Association of prebronchodilator and postbronchodilator spirometric measures with chronic bronchitis, dyspnoea scores, chest CT emphysema and air trapping and distance covered in 6 min walk test

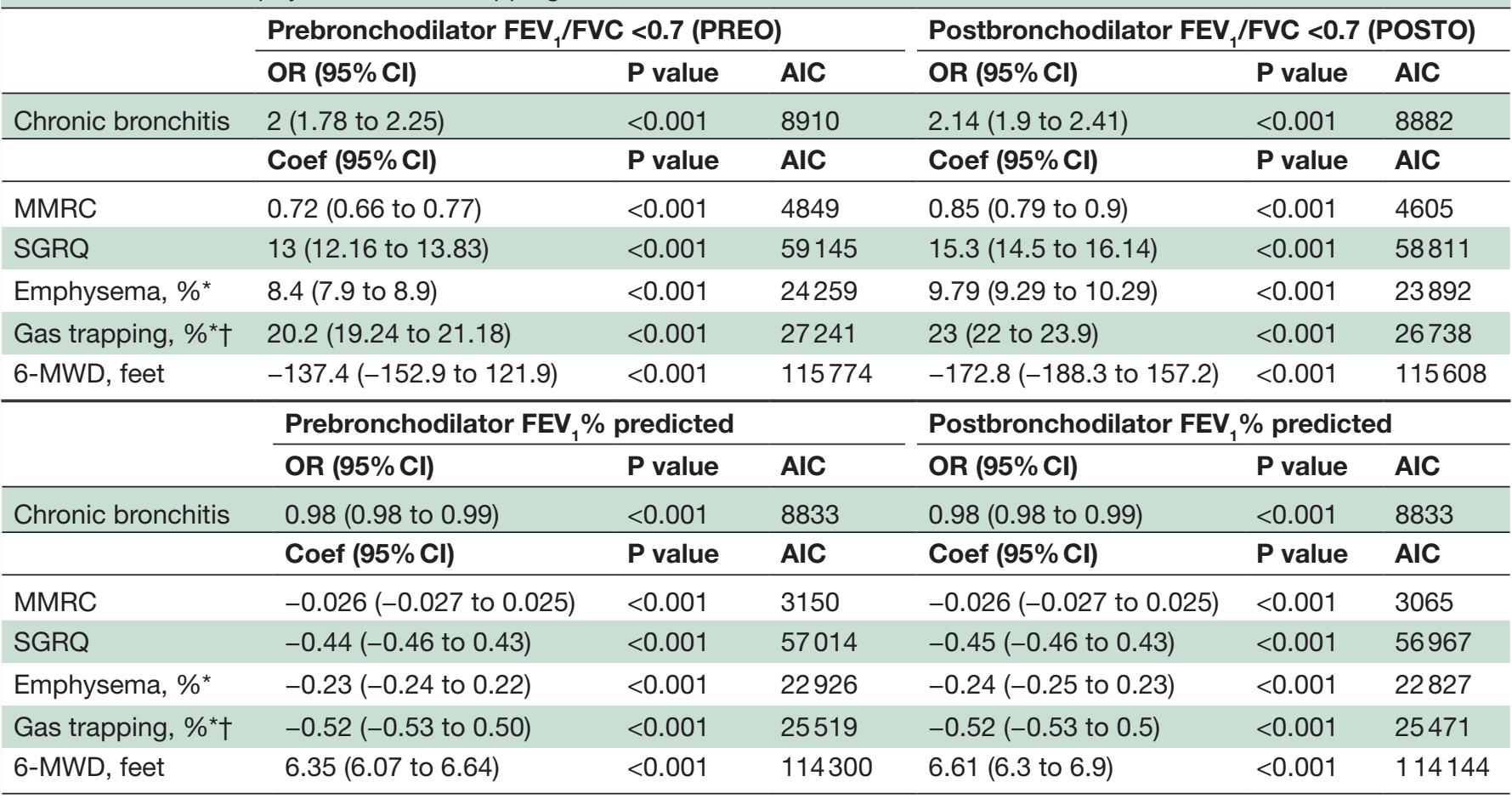

All regression models included the following covariates: age, sex, race, smoking status, pack per years, history (per questionnaire) of asthma, diabetes, high blood pressure, stroke and sleep apnoea.

${ }^{*}$ For emphysema and gas trapping analysis, data were available for 5553 and 4945 subjects, respectively.

†Gas trapping was measured at functional residual capacity.

6-MWD, 6 min walk distance; AIC, Akaike information criterion; FEV , forced expiratory volume in $1 \mathrm{~s}$; FVC, forced vital capacity; MMRC, modified Medical Research Council dyspnoea score; SGRQ, St. George's Respiratory Questionnaire score.

while $52.3 \%$ of those who progressed to PREN-POSTN were active smokers at the phase 2 visit $(\mathrm{P}=0.74)$.

\section{Mortality $(n=8221)$}

Subjects were followed in average for $1956 \pm 407$ days, and there were 830 deaths $(10.1 \%)$. All PREO, POSTO, pre-FEV $\%$, and post-FEV $\%$ were associated with mortality (table 5). Based on AIC, models that included post-FEV ${ }_{1} \%$ performed better at predicting mortality than the rest. Furthermore, PREO-POSTO was associated with increased mortality, while PREO-POSTN and PRENPOSTO were not (supplementary table 5).

\section{DISCUSSION}

In this report, $\mathrm{AFO}$ prevalence is higher when the PREO criterion is applied. About $8.5 \%$ of all subjects had PREO-POSTN, whereas $3 \%$ had a PREN-POSTO spirometric pattern. In adjusted analysis, we found no difference in COPD features and outcomes between PREOPOSTN and PREN-POSTO subjects. Although both prebronchodilator and postbronchodilator spirometry are associated with chronic bronchitis, dyspnoea, exercise capacity and COPD radiographic findings, models that include postbronchodilator spirometric measures perform better than those with prebronchodilator measures to predict those outcomes. The predictive value of prebronchodilator and postbronchodilator spirometries are relatively similar for respiratory exacerbations, change in $\mathrm{FEV}_{1}$ and dyspnoea from phase 1 to phase 2 visits. Both prebronchodilator and postbronchodilator spirometry are associated with mortality, but models that include postbronchodilator spirometric measures perform better than models with prebronchodilator spirometry. About half of PREO-POSTN and PREN-POSTN become PREO-POSTO at Phase 2. PREOPOSTO is associated with higher mortality compared with the other patterns.

The prevalence of AFO and therefore COPD is higher using prebronchodilator spirometry in our cohort, although previous studies have shown mixed results. ${ }^{718}$ Is this clinically significant? In the absence of a 'gold standard', a diagnostic test is good when it can predict outcomes and change management of a disease or condition. Both prebronchodilator and postbronchodilator spirometry were associated with chronic bronchitis, dyspnoea, radiographic emphysema and gas trapping and exercise capacity. Nevertheless, models that included postbronchodilator spirometric measures performed better compared with those that included postbronchodilator measures, indicating that postbronchodilator spirometry correlates better with those outcomes. 
Table 3 Number of exacerbations and severe exacerbations per year, change in FEV1, dyspnoea scores and distance covered in 6 min walk test from phase 1 to phase 2 visit (5-year interval)

\begin{tabular}{|c|c|c|c|c|c|c|}
\hline & $\begin{array}{l}\text { All } \\
(n=8479)\end{array}$ & $\begin{array}{l}\text { PREO-POSTO } \\
(\mathrm{n}=3619)\end{array}$ & $\begin{array}{l}\text { PREN-POSTN } \\
(\mathrm{n}=3936)\end{array}$ & $\begin{array}{l}\text { PREO-POSTN } \\
(\mathrm{n}=636)\end{array}$ & $\begin{array}{l}\text { PREN-POSTO } \\
(\mathrm{n}=288)\end{array}$ & P value* \\
\hline $\begin{array}{l}\text { Exacerbations per } \\
\text { year } \pm S D\end{array}$ & $0.42 \pm 0.99$ & $0.68 \pm 1.22$ & $0.21 \pm 0.70$ & $0.27 \pm 0.74$ & $0.26 \pm 0.79$ & 0.3 \\
\hline \multirow{2}{*}{$\begin{array}{l}\text { Severe exacerbations } \\
\text { per year } \pm S D\end{array}$} & $0.14 \pm 0.52$ & $0.24 \pm 0.66$ & $0.07 \pm 0.34$ & $0.1 \pm 0.5$ & $0.09 \pm 0.3$ & 0.069 \\
\hline & $\begin{array}{l}\text { All } \\
(n=4857)\end{array}$ & $\begin{array}{l}\text { PREO-POSTO } \\
\text { ( } n=1889)\end{array}$ & $\begin{array}{l}\text { PREN-POSTN } \\
(\mathrm{n}=2421)\end{array}$ & $\begin{array}{l}\text { PREO-POSTN } \\
(\mathrm{n}=379)\end{array}$ & $\begin{array}{l}\text { PREN-POSTO } \\
(n=168)\end{array}$ & P value* \\
\hline $\begin{array}{l}\text { Change in } \mathrm{FEV}_{1} \\
\mathrm{~mL} \pm \mathrm{SD}\end{array}$ & $-198.1 \pm 294.5$ & $-202.5 \pm 320.2$ & $-198.8 \pm 268.5$ & $-203.6 \pm 282.2$ & $-125.6 \pm 365.5$ & $<0.001$ \\
\hline $\begin{array}{l}\text { Change in MMRC } \\
\pm \text { SD }\end{array}$ & $0.06 \pm 1.24$ & $0.19 \pm 1.26$ & $-0.04 \pm 1.22$ & $0.03 \pm 1.08$ & $0.05 \pm 1.36$ & 0.42 \\
\hline $\begin{array}{l}\text { Change in SGRQ } \\
\pm S D\end{array}$ & $0.15 \pm 15.3$ & $1.5 \pm 15.2$ & $-0.8 \pm 15.4$ & $-0.88 \pm 14.6$ & $0.98 \pm 16$ & 0.86 \\
\hline $\begin{array}{l}\text { Change in 6-MWD, } \\
\text { feet } \pm S D\end{array}$ & $-130.9 \pm 360.6$ & $-172.7 \pm 366.4$ & $-103.7 \pm 357$ & $-103 \pm 327.4$ & $-123.5 \pm 377$ & $<0.001$ \\
\hline
\end{tabular}

${ }^{*}$ Comparison between PREO-POSTN and PREN-POSTO using $\chi^{2}$ and Student's t-test or Wilcoxon rank sum test.

6-6-MWD = 6-min walk distance; MMRC, modified Medical Research Council dyspnoea score; SGRQ, St. George's Respiratory

Questionnaire score.

PREN-POSTN: prebronchodilator $\mathrm{FEV}_{1} / \mathrm{FVC}>0.7$ and postbronchodilator $\mathrm{FEV}_{1} / \mathrm{FVC}>0.7$.

PREO-POSTNO: prebronchodilator $\mathrm{FEV}_{1} / \mathrm{FVC}<0.7$ and postbronchodilator $\mathrm{FEV}_{1} / \mathrm{FVC}<0.7$.

PREO-POSTN: prebronchodilator $\mathrm{FEV}_{1} / \mathrm{FVC}<0.7$ and postbronchodilator $\mathrm{FEV}_{1} / \mathrm{FVC}>0.7$.

PREN-POSTO: prebronchodilator $\mathrm{FEV}_{1} / \mathrm{FVC}>0.7$ and postbronchodilator $\mathrm{FEV}_{1} / \mathrm{FVC}<0.7$.

When we examined the subjects with AFO discordance in prebronchodilator and postbronchodilator spirometry, which comprised $11.7 \%$ of our total cohort, PRENPOSTO subjects have a remarkable increase in FVC after bronchodilator spirometry compared with PREO-POSTN subjects, although they have similar postbronchodilator FVC\%. PREN-POSTO subjects have likely more prebronchodilator air trapping than PREO-POSTN, which can be present in mild disease ${ }^{19} 20$ and result in exertional dyspnoea and lower exercise capacity. ${ }^{819} 21$ We did not observe higher radiographic air trapping in the PRENPOSTO than in the PREO-POSTN group, likely because CTs were performed after bronchodilator spirometry. When we adjusted for post-FEV ${ }_{1} \%$, PREN-POSTO was not associated with worse dyspnoea or exercise capacity compared with PREO-POSTN, which means that PRENPOSTO does not represent a different phenotype with increased air trapping but rather a group with more severe disease and lower post-FEV $\%_{1} .^{22}$

Prebronchodilator and postbronchodilator measures showed relatively similarly predictive value for long-term outcomes such as respiratory exacerbations, change of $\mathrm{FEV}_{1}$, dyspnoea score and exercise capacity. Previous studies have shown that bronchodilator response is associated with clinical outcomes, ${ }^{23}{ }^{24}$ but this association could be confounded by lung function. ${ }^{22}{ }^{25}$ For that reason, we adjusted for post-FEV $\%$ at baseline, and we found that the discordance groups had no difference in change of $\mathrm{FEV}_{1}$, dyspnoea score and exercise capacity.

Postbronchodilator spirometry models perform better than prebronchodilator spirometry models to predict mortality, although both are strongly associated with mortality. This is in disagreement with a previous study by Mannino et $a t^{6}$ that showed that both prebronchodilator and postbronchodilator spirometry can predict mortality with the same accuracy in a similar population to ours. Although subjects were followed for 15 years in their study instead of about 5 years in our study, their study was performed almost 20 years earlier than COPDGene, and they did not also include comorbidities in their analysis as we did. In AFO discordance groups, we did not find any difference in mortality between the two groups. PREOPOSTO subjects had increased mortality compared with the other groups, which may again reflect more advanced disease with lower post-FEV $\%{ }^{22}$ Although postbronchodilator may be superior to prebronchodilator spirometric measures to predict outcome, there are no available postbronchodilator spirometric reference values for a US population. Previous reports in Northern Europe $^{26}$ and South America ${ }^{27}$ have provided postbronchodilator spirometric reference values, but their predictive value for obstructive lung disease outcomes has not been compared with that of prebronchodilator reference values.

In addition, we showed that about $50 \%$ of the subjects with AFO discordance between prebronchodilator and postbronchodilator spirometry progress to PREOPOSTO, which is a pattern with higher mortality than the rest of the groups. Interestingly, subjects with PREOPOSTN that progressed to PREO-POSTO had higher smoking rates than those that progressed to PRENPOSTN in the follow-up visit; this raises the question 
Table 4 Association of prebronchodilator and postbronchodilator spirometric measures with number of exacerbations and severe exacerbations per year, change in FEV1, dyspnoea scores and distance covered in 6 min walk test from phase 1 to phase 2 visit (5-year interval)

\begin{tabular}{|c|c|c|c|c|c|c|}
\hline & \multicolumn{3}{|c|}{ Prebronchodilator FEV $/$ FVC $<0.7$ (PREO) } & \multicolumn{3}{|c|}{ Postbronchodilator FEV /FVC <0.7 (POSTO) } \\
\hline & Coef $(95 \% \mathrm{Cl})$ & $P$ value & AIC & Coef $(95 \% \mathrm{Cl})$ & $P$ value & AIC \\
\hline $\begin{array}{l}\text { Exacerbations per } \\
\text { year }\end{array}$ & 0.35 (0.31 to 0.4 ) & $<0.001$ & -893 & 0.38 (0.34 to 0.43$)$ & $<0.001$ & -929 \\
\hline $\begin{array}{l}\text { Severe } \\
\text { exacerbations per } \\
\text { year }\end{array}$ & 0.14 (0.11 to 0.16$)$ & $<0.001$ & -11608 & 0.14 (0.12 to 0.16$)$ & $<0.001$ & -11614 \\
\hline $\begin{array}{l}\text { Change in } \mathrm{FEV}_{1} \text {, } \\
\mathrm{mL}\end{array}$ & $-2.25(-20.3$ to 15.8$)$ & 0.81 & 54317 & $10(-7.26$ to 29.3$)$ & 0.24 & 54315 \\
\hline Change in MMRC & $0.2(0.12$ to 0.27$)$ & $<0.001$ & 1978 & 0.21 (0.13 to 0.29$)$ & $<0.001$ & 1974 \\
\hline Change in SGRQ & $1.92(0.99$ to 2.85$)$ & $<0.001$ & 26399 & 2.56 (1.62 to 3.5$)$ & $<0.001$ & 26387 \\
\hline $\begin{array}{l}\text { Change in } \\
\text { 6-MWD, feet }\end{array}$ & $-22.2(-44.3$ to 0.05$)$ & 0.049 & 55763 & $-33.2(-55.6$ to 10.8$)$ & 0.004 & 55758 \\
\hline
\end{tabular}

\begin{tabular}{|c|c|c|c|c|c|c|}
\hline & \multicolumn{3}{|c|}{ Postbronchodilator FEV $\%$ predicted } & \multicolumn{3}{|c|}{ Postbronchodilator $\mathrm{FEV}_{1} \%$ predicted } \\
\hline & Coef $(95 \% \mathrm{Cl})$ & $P$ value & AIC & Coef $(95 \% \mathrm{Cl})$ & $P$ value & AIC \\
\hline Exacerbations & $-0.011(-0.012$ to 0.01$)$ & $<0.001$ & -1233 & $-0.011(-0.01$ to 0.0097$)$ & $<0.001$ & -1222 \\
\hline $\begin{array}{l}\text { Severe } \\
\text { exacerbations }\end{array}$ & $-0.004(-0.0045$ to 0.0036$)$ & $<0.001$ & -11787 & $-0.004(-0.0045$ to 0.0036$)$ & $<0.001$ & -11777 \\
\hline $\begin{array}{l}\text { Change in } \mathrm{FEV}_{1} \text {, } \\
\mathrm{mL}\end{array}$ & $-1.91(-2.28$ to 1.53$)$ & $<0.001$ & 54218 & $-2.76(-3.13$ to 2.39$)$ & $<0.001$ & 54112 \\
\hline Change in MMRC & $-0.002(-0.0038$ to 0.00065$)$ & 0.006 & 1996 & $-0.02(-0.0036$ to 0.00037$)$ & 0.016 & 1998 \\
\hline Change in SGRQ & $-0.01(-0.03$ to 0.0095$)$ & 0.32 & 26415 & $-0.01(-0.029$ to 0.01$)$ & 0.34 & 26415 \\
\hline $\begin{array}{l}\text { Change in } \\
6-M W D \text {, feet }\end{array}$ & 0.99 (0.53 to 1.46$)$ & $<0.001$ & 55749 & 0.9 (0.43 to 1.37$)$ & $<0.001$ & 55753 \\
\hline
\end{tabular}

All regression models included the following covariates: age, sex, race, smoking status, pack per years, history (per questionnaire) of asthma, diabetes, high blood pressure, stroke and sleep apnoea.

6-MWD, 6 min walk distance; AIC, Akaike information criterion; FEV , forced expiratory volume in $1 \mathrm{~s}$; FVC, forced vital capacity; MMRC, modified Medical Research Council dyspnoea score; SGRQ, St. George's Respiratory Questionnaire score.

whether this group represents patients with early disease to whom we need to target interventions like smoking cessation. This finding suggest that using prebronchodilator spirometry may be a better diagnostic test for early (or future) disease although postbronchodilator spirometry correlates better with current symptoms, functional and radiographic measures and 5-year mortality.

\begin{tabular}{|c|c|c|c|}
\hline & HR $(95 \% \mathrm{Cl})$ & $P$ value & AIC \\
\hline PREO & 2.46 (2.07 to 2.92$)$ & $<0.001$ & 14194 \\
\hline POSTO & 2.54 (2.15 to 3 ) & $<0.001$ & 14181 \\
\hline Pre-FEV ${ }_{1} \%$ & 0.97 (0.96 to 0.97$)$ & $<0.001$ & 13837 \\
\hline Post-FEV $\%$ & 0.97 (0.96 to 0.97$)$ & $<0.001$ & 13806 \\
\hline
\end{tabular}

All regression models included the following covariates: age, sex, race, smoking status, pack per years, history (per questionnaire) of asthma, diabetes, high blood pressure, stroke and sleep apnoea. AIC , Akaike information criterion; POSTO, postbronchodilator $\mathrm{FEV}_{1} / \mathrm{FVC}<0.7$; pre- $\mathrm{FEV}_{1} \%$, prebronchodilator $\mathrm{FEV}_{1} \%$ predicted; post-FEV $\%$, postbronchodilator $\mathrm{FEV}_{1} \%$ predicted; PREO, prebronchodilator $\mathrm{FEV}_{1} / \mathrm{FVC}<0.7$.
Apart from the fact that chest CTs were performed after bronchodilator and gas trapping was measured at FRC our study is limited by the large variability of bronchodilator response. Although, we used albuterol and the same protocol for all the bronchodilations, greater bronchodilator response may occur when spirometric manoeuvres were performed $>20$ min after bronchodilator administration instead of $15-20$ min. ${ }^{22} 28$ Subjects older than 80 years were not included. We only have phase 2 spirometries for half of the subjects. The follow-up period may not be long enough to detect some outcome differences, especially mortality, between the AFO discordance groups. We do not have data on the specific cause of death. We did not also detect any robust outcome differences, as both prebronchodilator and postbronchodilator spirometries were associated strongly with outcomes. Postbronchodilator spirometry superiority is based on better model performance using the AIC. ${ }^{16}{ }^{17}$ These limitations do not undermine the strengths of our study, which include the large sample size and the wealth of epidemiological data.

In conclusion, PREO was more sensitive to diagnose AFO compared with POSTO. About half of the subjects 
with AFO discordance in their prebronchodilator and postbronchodilator spirometry, which compromise $11 \%$ of all subjects, progress to PREO-POSTO, which is a pattern with higher mortality compared with the other patterns. Although both prebronchodilator and postbronchodilator spirometries are associated with clinical, functional and radiographic features of COPD, and mortality, our findings suggest that postbronchodilator spirometry may be a more accurate measure of COPD burden and should be used for COPD diagnosis and classification. This raises the question of whether postbronchodilator spirometric reference values for the US population are needed.

Acknowledgements We thank Paul Casella, MFA for editorial assistance and PatrickTen Eyck, PhD for statistical consult.

Collaborators COPDgene investigators

Contributors All authors made substantial contributions to the study. SF participated in study conception and design, data analysis and interpretation and drafting of the manuscript. ME participated in study design, data interpretation and drafting of the manuscript. DG participated in data interpretation. AC participated in study conception and design, data interpretation and drafting of the manuscript.

Funding The project described was supported by Award Number R01 HL089897 and Award Number R01 HL089856 from the National Heart, Lung, and Blood Institute. The COPDGene project is also supported by the COPD Foundation through contributions made to an Industry Advisory Board comprised of AstraZeneca, Boehringer Ingelheim, GlaxoSmithKline, Novartis, Pfizer, Siemens and Sunovion.

Competing interests None declared.

Ethics approval The institutional review boards at each participating center outlined below approved the study protocol. Details of the study protocol have been published previously. ${ }^{12}$

Provenance and peer review Not commissioned; externally peer reviewed.

Data sharing statement Please contact COPD gene investigators for additional data request.

Open Access This is an Open Access article distributed in accordance with the Creative Commons Attribution Non Commercial (CC BY-NC 4.0) license, which permits others to distribute, remix, adapt, build upon this work non-commercially, and license their derivative works on different terms, provided the original work is properly cited and the use is non-commercial. See: http://creativecommons.org/ licenses/by-nc/4.0/

(C) Article author(s) (or their employer(s) unless otherwise stated in the text of the article) 2017. All rights reserved. No commercial use is permitted unless otherwise expressly granted.

\section{REFERENCES}

1. Global initiative for chronic obstructive lung disease. Guide to copd diagnosis, management, and prevention. http://goldcopd.org/gold2017-global-strategy-diagnosis-management-prevention-copd/

2. Vaz Fragoso CA, Concato J, McAvay G, et al. Chronic obstructive pulmonary disease in older persons: A comparison of two spirometric definitions. Respir Med 2010;104:1189-96.

3. Mannino DM, Diaz-Guzman E. Interpreting lung function data using $80 \%$ predicted and fixed thresholds identifies patients at increased risk of mortality. Chest 2012;141:73-80.

4. Vaz Fragoso CA, Concato J, McAvay G, et al. The ratio of FEV1 to FVC as a basis for establishing chronic obstructive pulmonary disease. Am J Respir Crit Care Med 2010;181:446-51.
5. Chen CZ, Ou CY, Wang WL, et al. Using post-bronchodilator FEV is better than pre-bronchodilator $\mathrm{FEV}_{1}$ in evaluation of COPD severity. COPD 2012:9:276-80.

6. Mannino DM, Diaz-Guzman E, Buist S. Pre- and post-bronchodilator lung function as predictors of mortality in the Lung Health Study. Respir Res 2011;12:136.

7. Tilert T, Dillon C, Paulose-Ram R, et al. Estimating the U.S. prevalence of chronic obstructive pulmonary disease using pre- and post-bronchodilator spirometry: the National Health and Nutrition Examination Survey (NHANES) 2007-2010. Respir Res 2013;14:103.

8. O'Donnell DE. Hyperinflation, dyspnea, and exercise intolerance in chronic obstructive pulmonary disease. Proc Am Thorac Soc 2006;3:180-4.

9. Pellegrino R, Viegi G, Brusasco V, et al. Interpretative strategies for lung function tests. Eur Respir J 2005;26:948-68.

10. Newton MF, O'Donnell DE, Forkert L. Response of lung volumes to inhaled salbutamol in a large population of patients with severe hyperinflation. Chest 2002;121:1042-50.

11. Fortis S. Lost in interpretation: should the highest VC value be used to calculate the FEV1/VC ratio? Int J Chron Obstruct Pulmon Dis 2016;11:2167-70.

12. Regan EA, Hokanson JE, Murphy JR, et al. Genetic epidemiology of COPD (COPDGene) study design. COPD 2010;7:32-43.

13. Miller MR, Hankinson J, Brusasco V, et al. Standardisation of spirometry. Eur Respir J 2005;26:319-38.

14. Hankinson JL, Odencrantz JR, Fedan KB. Spirometric reference values from a sample of the general U.S. population. Am J Respir Crit Care Med 1999;159:179-87.

15. Wan ES, Hokanson JE, Murphy JR, et al. Clinical and radiographic predictors of gold-unclassified smokers in the COPDgene study. Am J Respir Crit Care Med 2011;184:57-63.

16. Burns RJ, Deschênes SS, Schmitz N. Associations between depressive symptoms and social support in adults with diabetes: Comparing directionality hypotheses with a longitudinal cohort. Ann Behav Med 2016;50:348-57.

17. Spolverato G, Ejaz A, Kim Y, et al. Prognostic performance of different lymph node staging systems after curative intent resection for gastric adenocarcinoma. Ann Surg 2015;262:991-8.

18. Pérez-Padilla R, Hallal PC, Vázquez-García JC, et al. Impact of bronchodilator use on the prevalence of COPD in population-based samples. COPD 2007;4:113-20.

19. Fortis S, Corazalla EO, Kim HJ. Does normal spirometry rule out an obstructive or restrictive ventilatory defect? Respir Investig 2017;55:55-7.

20. O'Donnell DE, Deesomchok A, Lam YM, et al. Effects of BMI on static lung volumes in patients with airway obstruction. Chest 2011;140:461-8.

21. Foglio K, Carone M, Pagani M, et al. Physiological and symptom determinants of exercise performance in patients with chronic airway obstruction. Respir Med 2000;94:256-63.

22. Calverley PM, Albert P, Walker PP. Bronchodilator reversibility in chronic obstructive pulmonary disease: use and limitations. Lancet Respir Med 2013;1:564-73.

23. Scanlon PD, Connett JE, Waller LA, et al. Smoking cessation and lung function in mild-to-moderate chronic obstructive pulmonary disease. The Lung Health Study. Am J Respir Crit Care Med 2000;161:381-90.

24. Vestbo J, Edwards LD, Scanlon PD, et al. Changes in forced expiratory volume in 1 second over time in COPD. N Engl J Med 2011;365:1184-92

25. Albert P, Agusti A, Edwards L, et al. Bronchodilator responsiveness as a phenotypic characteristic of established chronic obstructive pulmonary disease. Thorax 2012;67:701-8.

26. Johannessen A, Lehmann S, Omenaas ER, et al. Post-bronchodilator spirometry reference values in adults and implications for disease management. Am J Respir Crit Care Med 2006;173:1316-25.

27. Pérez-Padilla R, Torre Bouscoulet L, Vázquez-García JC, et al. Spirometry reference values after inhalation of 200 microg of salbutamol. Arch Bronconeumol 2007;43:530-4.

28. Campbell S. For COPD a combination of ipratropium bromide and albuterol sulfate is more effective than albuterol base. Arch Intern Med 1999;159:156-60. 